
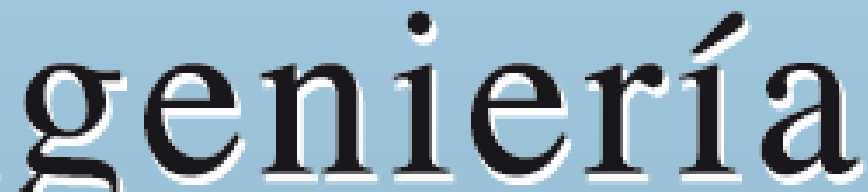

Revista de la Universidad de Costa Rica ENEROIDICIEMBRE 2002 - VOLUMEN 12 - $\mathrm{N}^{\circ} 1$ y 2

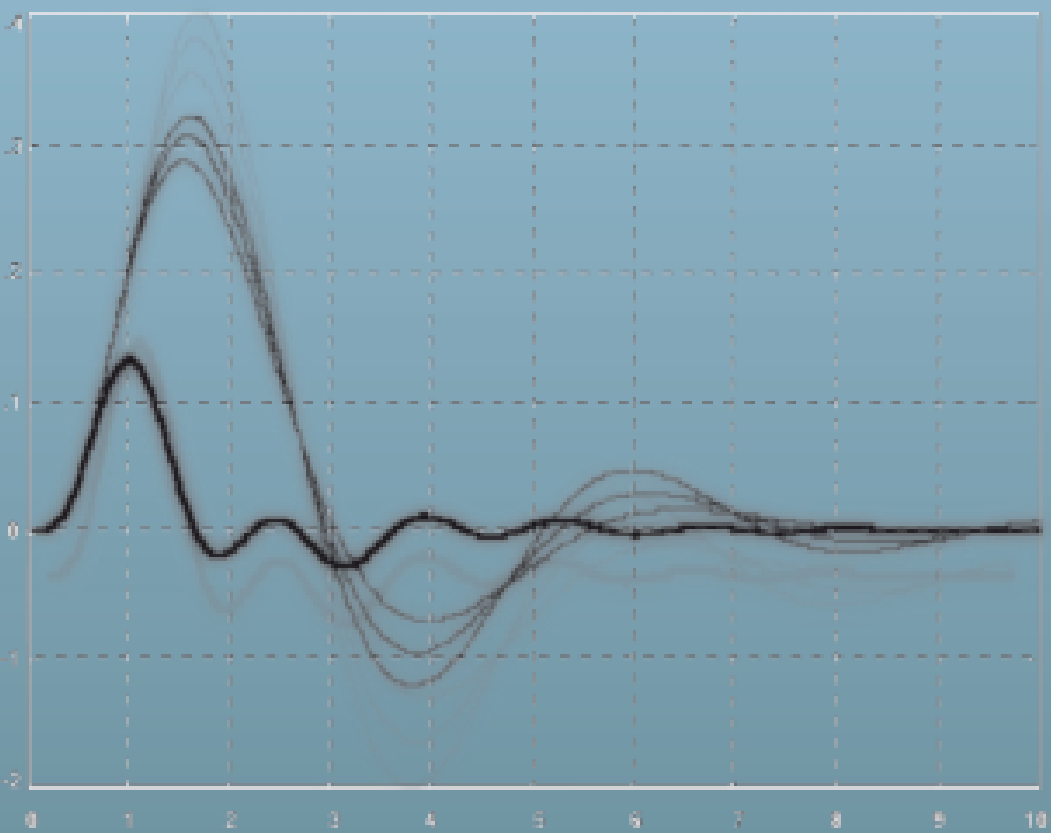




\title{
ACCIONAMENTO DIRECTO DE HERRAMIENTAS DE CORTE EN MÁQUINAS DE ULTRA-PRECISIÓN
}

\author{
Luis Arturo Rapso Brenes \\ Juan Carlos Campos Rubio
}

\begin{abstract}
Resumen
En el campo de la ingeniería de precisión, el desempeño adecuado de las máquinas-herramienta depende de las características dinámicas de los sistemas de accionamiento. La mecanización de piezas de formas complejas con reducidos errores dimensionales y de contorno (nanómetros), pequeñas profundidades de corte y acabados superficiales satisfactorios (ángstrom), entre otros, es difícil de alcanzar con servo accionamientos rotativos convencionales. Este trabajo corresponde a un estudio preliminar sobre la utilización del accionamiento directo de herramientas de corte a través de actuadores lineales (servomotores translativos). Mostramos y discutimos los diversos tipos de actuadores lineales que pueden servir de base para el diseño de una máquina-herramienta de ultra-precisión
\end{abstract}

Palabras clave: motores translativos, actuadores lineales, máquinas de ultra-precisión, servo accionamientos.

\begin{abstract}
In the precision engineering grounds, the adequate performance of machine tools depends upon the characteristics of the built-in positioning systems. The machining of complex shapes, with low dimensional and contour errors (of the order of nanometers), small depths of cut, optical grade surface texture is very difficult to be accomplished with conventional rotational servos, calling for the use of direct driving of the cutting tool through linear actuators. This paper presents a study of the performance of several types of linear actuators as a basis for the design of precision machine tools.
\end{abstract}

Keywords: linear motors, linear actuators, precision machines, servomechanisms, micro positioning systems.

\section{INTRODUCCIÓN}

La competitividad en la industria moderna exige cada vez más productos de alta calidad con tolerancias ajustadas y formas complejas, lo que se hace sentir en cada etapa de su diseño. De esta forma, y como respuesta al grado de precisión dimensional y geométrica exigida a los productos, las máquinas-herramienta están en permanente modificación.

El sistema más utilizado para el accionamiento de posicionadores en las máquinas-herramienta $\mathrm{CNC}$ es el constituido por un servomotor rotativo acoplado a un conjunto tornillo/tuerca de esferas recirculantes a través de un elemento flexible para la absorción de los desalineamientos. (Figura 1.)

Lo anterior presenta algunas desventajas propias de este tipo de configuración, por ejemplo:
- Presencia de holguras

- Incremento de la inercia

- No linealidades (histéresis, fricción)

- Menor velocidad crítica, etc.

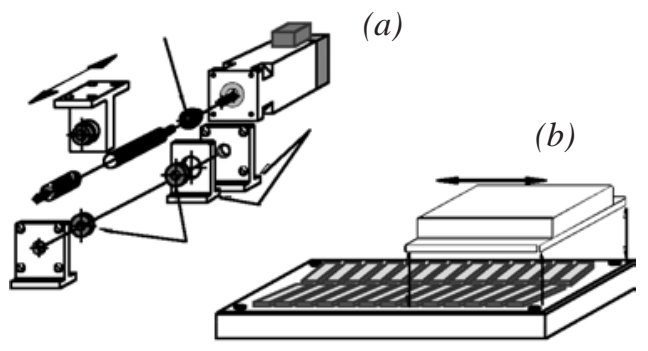

Figura 1. Componentes básicos de un sistema posicionador utilizando Servomotores (a) Rotativos y

(b) Translativos 
En el campo de la Ingeniería de Precisión estas dificultades se acentúan, por lo tanto, se estudian alternativas que permitan reducir significativamente dichos inconvenientes, a saber, motores lineales.

\section{SERVOMOTORES EN MÁQUINAS-HERRAMIENTA}

El motor es el elemento que realiza una conversión de señales y energías entre el sistema eléctrico y el mecánico, asi como también del sistema eléctrico en energía térmica y ruido.

Rapidez de movimiento, repetibilidad, velocidades máximas y mínimas constantes, facilidad de control, confiabilidad y economía son objetivos que han orientado el desarrollo de las máquinas eléctricas para la aplicación en máquinas-herramienta y robótica, y en conjunto han llevado a diversas concepciones no sólo de motores eléctricos sino de sistemas de energía y sistemas de control.

La aparición y evolución de los servomotores coincide con la constante búsqueda de alto

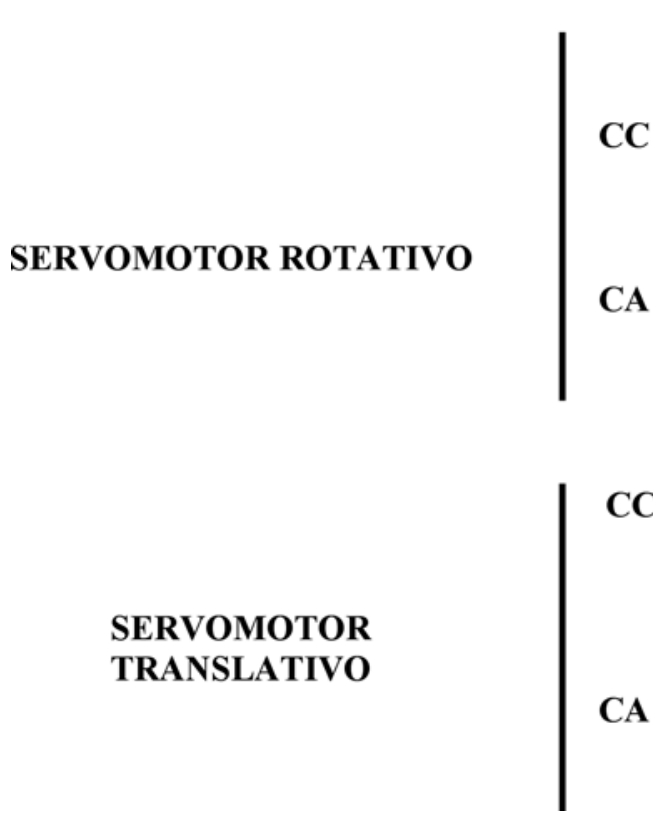

CC desempeño y la eliminación de los factores limitantes. El servomotor se distingue de los "motores comunes" por el hecho de que estos últimos son optimizados en función del mínimo costo, alta eficiencia, larga vida útil, etc., mientras que los servomotores en función de su desempeño estático y principalmente de su dinámica. De esta forma se puede decir que los servomotores son aquellos que pueden ser controlados en posición y/o velocidad y que responden a las órdenes recibidas con elevada rapidez y precisión (Campos Rubio, 1992)

Considerando el tipo de movimiento mecánico (rotación o translación) resultante, los servomotores utilizados en máquinas controladas automáticamente pueden ser clasificados, dentro de este contexto, según el esquema mostrado en la Figura 2.

Un grupo especial es el constituido por los actuadores piezoeléctricos, cuyo principio de funcionamiento está basado en fenómenos de estricción electrostática, sin embargo, no serán analizados en este artículo.

\section{SERVOMOTOR}

con escobillas sin escobilla (brushless ) sin núcleo (coreless, ironless ) de paso

síncronos asíncronos (de inducción) sin escobillas ( BL Senoidal)

$$
\mid \begin{aligned}
& \text { bobina móvil (moving- } \\
& \text { coil, voice-coil } \\
& \text { de paso }
\end{aligned}
$$

$\mid \begin{aligned} & \text { sincrónicos } \\ & \text { asíncronos } \\ & \text { (de inducción })\end{aligned}$

Figura 2. Clasificación de servomotores utilizados en máquinas controladas automáticamente. 
Un grupo especial es el constituido por los piezoelélctricos, cuyo principio de funcionamiento es basado en fenomenos de estricción electrostática. Estos actuadores no serán analizados en este documento.

\section{SERVOMOTORES LINEALES O TRANSLATIVOS}

Como puede observarse en la Figura 1, parte b, al contrario de los servomotores rotativos, los motores lineales eliminan acoplamientos y conversores de movimiento permitiendo desplazamientos de translación exentos de errores provenientes de la histéresis de los tornillos, las cadenas de transmisión y holguras por los acoplamientos, engranajes y correas.

Una diferencia importante entre los motores rotativos y los lineales consiste en que los primeros producen una fuerza tangencial al par de salida, mientras que en los motores lineales se obtiene una fuerza resultante.

\subsection{Servomotores lineales de pasos}

\section{(Lineal Step Motors)}

Concebidos a partir del motor de pasos rotativo, poseen un cursor que desempeña el papel del rotor en el motor rotativo. constituye un cuerpo plano que contiene imanes permanentes. El estator es de material ferromagnético semejante al del motor de pasos rotativo, sin embargo, es de forma plana y cierra el circuito magnético.

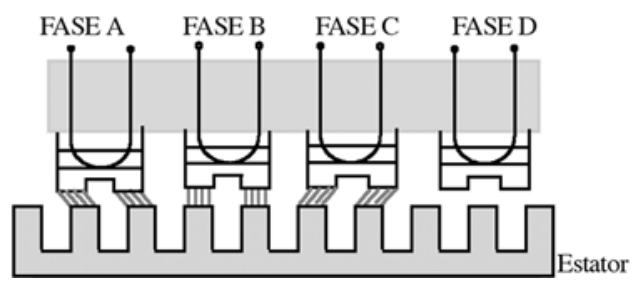

Figura 3. Motor de pasos lineal.

Los motores lineales de pasos convierten energía eléctrica, controlada por señales digitales de comando, en movimientos de translación discretos (discontinuos), con pasos completos que toman valores de $5 \mu \mathrm{m}$, aproximadamente, y una fuerza máxima de desplazamiento superior a los $4000 \mathrm{~N}$. $\mathrm{El}$ cursor se caracteriza por presentar un paso entre los dientes diferente del paso del estator.

La utilización de los motores de pasos lineales en sistemas de posicionamiento de alta precisión se encuentra todavía en fase de investigación. Por otra parte, los sistemas de posición que utilizan este tipo de accionamiento requieren sensores de posición.

Al igual que el motor de pasos rotativo, el motor de pasos lineal es capaz de posicionar pequeñas cargas sin necesidad de realimentación. Sin embargo, para posicionamientos sub-micrométricos, debido a la variación de la fuerza de translación (picos de intensidad), cogging, es aconsejable utilizar sensores de posición tales como escalas opto electrónicas o láser interferométrico.

De esta forma, si se utilizan motores controlados electrónicamente en posicionadores, linear microstepping motors, es posible alcanzar resoluciones inferiores a 0,1 $\mu \mathrm{m}$ (Lammers, 1994); sin obviar el hecho de que el uso de amplificadores de micropaso reducen notablemente la fuerza de retención.

\subsection{Servotores lineales sin escobillas (Brusheless Linear Motors)}

Los servomotores de corriente continua brushless o "sin escobillas" funcionan de manera semejante a su análogo rotativo. Están constituidos, básicamente, por un cursor con imanes permanentes y un estator electromagnético, embobinados en el estator. La conmutación de las bobinas se realiza electrónicamente, el campo electromagnético generado interactúa con el campo magnético de los imanes permanentes del cursor. De ello resulta una fuerza de translación proporcional a la intensidad de la corriente (Fuerza de Lorentz).

Existen esencialmente dos tipos de servomotores lineales sin escobillas, los de cursor ferromagnético y los de cursor no magnético (aluminio, epoxi). 
Los motores con cursor de material ferromagnético se caracterizan por poseer una alta densidad de flujo magnético y consecuentemente una mayor fuerza resultante; sin embargo, suelen ser pesados y voluminosos con variaciones en la fuerza de hasta $5 \%$ del valor medio resultante. Además, por ser ferromagnéticos, surge un componente normal al movimiento de la carga, la cual cuando es excesiva puede causar errores de rectilineidad y planicidad en las guías así como movimientos extrínsecos de la carga (Lammers, 1994).

Por otro lado, en los motores lineales sin escobillas con cursor no magnético, tanto el componente normal como los picos o variaciones de fuerza, cogging, son nulos. Por ende, se consideran más adecuados para los regímenes de alta y baja velocidad de translación.

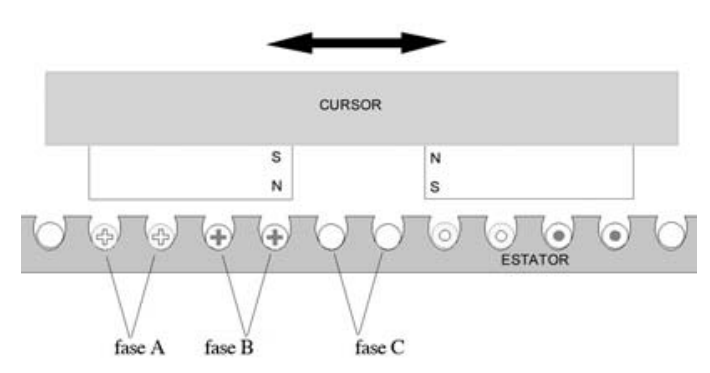

Figura 4. Motor CC Lineal tipo "Brushless" ( tres fases con imanes móviles).

\section{COMENTARIOS Y SUGERENCIAS}

Varios investigadores han optado por soluciones que utilizan servo-sistemas dotados de doble mecanismo de posicionamiento. Para ello combinan un sistema de posicionamiento grosero vía servomotor rotativo y un sistema de posicionamiento fino con actuador lineal, así como un algoritmo de control adecuado y secuencial como en Tavares (1995) y Miller et al (1994) o continuo/combinado como en Okazaki (1993), este último ha obtenido resoluciones del orden de $5 \mathrm{~nm}$.

Otros estudios experimentales apuntan hacia la utilización de accionamientos de fricción como sistema primario (Sakuta et al, 1993), así como a la utilización de motores tipo bobina móvil, solución viable y de resultados que entusiasman.
Una solución alternativa sería solamente utilizar un motor translativo de alto desempeño y elevar su comportamiento a través de algoritmos de control y corrección adecuados, como en Rojas Vázquez (1994) y Tomita et al (1994).

Es necesario resaltar que aunque la resolución alcanzada en el posicionamiento de máquinas de ultra-precisión sea consecuencia directa del tipo de accionamiento adoptado y de su desempeño dinámico, factores como rozamiento de las guías, temperatura, ruidos y vibraciones en el entorno, algoritmos de control adoptado, entre otros, juegan un papel extremamente importante en el resultado final (Weingaertner, 1992).

Para finalizar se puede afirmar que al seleccionar entre un motor translativo y un actuador lineal, el curso máximo permitido, la dinámica de respuesta, la facilidad de control y la capacidad de carga, en orden de importancia, representan variantes que deben ser avaladas concienzudamente.

\section{AGRADECIMIENTOS}

Los autores agradecen al CNPq de Brasil por el apoyo financiero concedido.

\section{BIBLIOGRAFÍA}

1. Campos Rubio, J. C.; Controladora Flexivel Multi-Eixo para Acionamentos Fracionários de Alta Dinâmica, Dissertación de Mestrado, UFSC,1995

2. Etrema Products, Inc.; Etrema Terfenold-D® Magnetostriction, (catalog), 1994, IA, USA.

3. Gerchman, M. et Youden, D.; An Evaluation elf Ultra-precise Machine Tool Contouring Performance: The Low Amplitude Sine Tracking (LAST) Test. Progress in Precision Engineering, Springer-Verlag, Heidelberg, 1991.

4. Izuno, Y. et al ; Fuzzy Reasoning-Based HighPerformance Speed/Posit Ulttrasonic Motor. IEEE - Transaction eln Industry Aplication, Vol. 28, №3, 1992. 
5. Itao, K.; Precision Mectemisms for Mechatronics Systems. Int. J. Japan Soc. Prec. Eng., Vol. 27, N4, 1993.

6. Kim, J. et Nam S.; An Improvement elf Positionig Accuracy by Use elf Piezoelectric Voltage in Piezoelectric Driven Micropositioning System Simulation, Mech. Mach. Theory, Vol. 30/6/ 1995.

7. Lammers, M.G.; Lineal Lead in Ultrasmooth Motion, Machine Desing, Vol. 17, 1994, USA.

8. Machine Desing Magazine; Basics elf Desing Engineering - Motion Control / Components, Reference Volume, april, 1995.

9. Miller, M. et al; A Controller Architecture for Integrating a Fast Tool Servo into a Diamond Turning Machine. Precision Engineering, Vol. 16, N¹, 1994.

10. Okazaki, Y. et al; Dual Servo Mectemical Stage for Continuous Positioning. Int. J. Japan Soc. Prec. Eng., Vol. 27, №2, 1993.

11. Pritschow, G. et Philipp W. ; Direct Drives for High-Dynamics Machine Tool Axes, Annals elf the CIRP, Vol. 39/1/1990.

12. Pfeifer, T.; Elektrische Messung mectemischer Größen, Tutorial, WZL T. H., Aachen, 1981.

13. Phisik Instrumente; Products for micropositionig, (catalog), 1992, Germany.

14. Tavares, R. C.; Projeto de un Posicionador Sub-Micrométrico para Litografia Óptica, Dissertación de Mestrado, UFSC,1995.

15. Tomita, Y. et al; A Surface Motor-Drive Precise Positioning System. Precision Engineering, Vol. 16, N³, 1994.

16. Rojas; Vázquez, J.; Desarrollo de un Posicionador de Ultra-Precisào, Dissertação de Mestrado, UFSC,1994.

17. Sakuta, S. et al; Experimental Studies in Ultra-Precision Positioning. Int. J. Japan Soc. Prec. Eng., Vol. 27, N³, 1993.
18. Sánchez, L. et al.; Sistema Ativo de Estabilização Aplicado à Holografía Eletrônica, Anales VI C.I.M., Santiago,1994

19. Shaw, M. C.; Precision Finishing, Annals elf the CIRP., Vol. 44/1/1995.

20. Slocum, A. H.; Precision Machine Desing, Printice-Hall, Inc., 1992, USA.

21. Weingaertner, W. L.; Desarrollos en la Usinagem de Ultraprecison, Monográfia, UFSC, 1992

22. Wang, X.; Experimental Research eln the Lineal Motor Micro-Device with HighFrequency Response, Long Travel and High Accuracy. Annais elf the CIRP, Vol. 40/1/1991.

23. Weck, M.et al; Analyzing the Dynamics Performance elf High Precision Machine, Tutorial, IPT, Aachen, 1991.

24. Weck, M. et Bispink, T.; Examination elf High Precision Slow-motion Feed-drive Systems for the Sub-micrometre Range. Progress in Precision Engineering, Springer-Verlag, Heidelberg, 1991.

25. Zhou, S-W.; An Magnetostrictive Mount For Isolating Base Vibration - Part I. XIII COBEM/II CIDIM, Belo Horizonte, 1995.

\section{SOBRE LOS AUTORES}

\section{Luis Arturo Rapso Brenes}

Profesor Escuela de Ingeniería Mecánica. Universidad de Costa Rica.

Email:lrapso@cariari.ucr.ac.cr

\section{Juan Carlos Campos Rubio}

Profesor Escuela de Ingeniería Mecánica. Universidad Federal de Minas Gerais, Belo Horizonte, Brasil.

Email: juan@vesper.demec.ufmg.br 
